\title{
Caracterización de los departamentos de la región Caribe utilizando los indicadores de logros educativos del DNP
}

\section{Caracterização dos departamentos da região das Caraíbas utilizando os indicadores de desempenho escolar da DNP}

DOI: $10.54021 /$ seesv3n1-011

Recebimento dos originais: 05/12/2021

Aceitação para publicação: 27/01/2022

\section{Andrea Carolina Hernández Márquez}

Licenciada en Matemáticas, Universidad de Sucre

Universidad de Sucre- Grupo de investigación Estadística y Modelamiento

Matemático Aplicado a Calidad Educativa.

Cra 28 \# 5-267 Barrio Puerta Roja - Sincelejo (Sucre). Bloque 5-Salon 106.

E-mail: ancarohernandez@gmail.com

\section{María Clareth Méndez Ramos}

Especialista en Estadística Aplicada, Fundación Universitaria Los Libertadores.

Universidad de Sucre- Grupo de investigación Estadística y Modelamiento Matemático Aplicado a Calidad Educativa.

Cra 28 \# 5-267 Barrio Puerta Roja - Sincelejo (Sucre). Bloque 5-Salon 106.

E-mail: mariaclare29@gmail.com

\section{Andrea Carolina Menco Tovar}

Licenciada en Matemáticas, Universidad de Sucre

Universidad de Sucre- Grupo de investigación Estadística y Modelamiento Matemático Aplicado a Calidad Educativa.

Cra 28 \# 5-267 Barrio Puerta Roja - Sincelejo (Sucre). Bloque 5-Salon 106.

E-mail: andreacarolinamenco1996@gmail.com

\section{Melba Liliana Vertel Morinson}

Magister en Ciencias Estadísticas, Universidad Nacional de Colombia.

Docente titular. Universidad de Sucre- Líder del Grupo de investigación

Estadística y Modelamiento Matemático Aplicado a Calidad Educativa.

Cra 28 \# 5-267 Barrio Puerta Roja - Sincelejo (Sucre). Bloque 5-Salon 106.

E-mail: melba.vertel@unisucre.edu.co

\section{RESUMEN}

El objetivo principal de esta investigación fue caracterizar los departamentos de la región Caribe en Colombia haciendo uso de indicadores de logro educativo como lo es la asistencia escolar. Ello por medio de un estudio de tipo descriptivo, para el cual se implementó la metodología del Análisis de Componentes principales a las variables de indicadores de logos educativos proporcionados por el Departamento Nacional de Planeación-DNP, por medio de esta técnica se presenta una descripción específicamente del comportamiento de las variables que dan respuesta al problema de investigación. En términos de los resultados 
se evidencio que la región Caribe presentó los mejores niveles de asistencia escolar la presentan Sucre, Magdalena y Bolívar. En donde el grupo de la población de jóvenes entre los 18 y 24 años es la que presenta los menores niveles de asistencia escolar.

Palabras Claves: Educación, logros, Indicadores, Análisis de Componentes Principales.

\section{ABSTRACT}

The main objective of this research was to characterize the departments of the Caribbean region in Colombia using educational achievement indicators such as school attendance. This was done by means of a descriptive study, for which the methodology of Principal Component Analysis was implemented to the variables of educational achievement indicators provided by the National Planning Department-DNP, by means of this technique a specific description of the behavior of the variables that respond to the research problem is presented. In terms of the results, it is evident that the Caribbean region presented the best levels of school attendance in Sucre, Magdalena and Bolivar. The group of young people between 18 and 24 years of age is the one with the lowest levels of school attendance.

Keywords: Education, achievement, indicators, Principal Component Analysis.

\section{INTRODUCCIÓN}

En Colombia en las últimas décadas el sistema educativo ha presentado cambios, derivados de la puesta en marcha de políticas públicas que se han enfocado en ampliar y mejorar la cobertura, calidad y eficiencia educativa; sin embargo, a pesar de los esfuerzos realizados la educación en el país sigue presentado indicadores poco consistentes y eficientes que indican que en la realidad son múltiples los factores que afectan la estabilidad y el mejoramiento de la educación (García, 2005)

Por su parte la región Caribe ha presentado avances en esta materia, sin embargo los factores sociales desencadenantes de la violencia y el mal manejo de las políticas, los indicadores no mejoran en las proporciones en las que se esperaría, es por ello que la presente investigación nace de la necesidad de caracterizar la situación y el comportamiento de los indicadores de logro educativo con el fin de evaluar y comparar los resultados a nivel de país y haciendo especial énfasis en la Región Caribe (Terrádez, 2010; Viloria, 2006).

Así mismo algunos antecedentes relacionados con en este estudio son:

En primera instancia el trabajado desarrollado por (André Lestage, 1982), el cual es un artículo relacionado con analfabetismo y alfabetización, publicado 
por la UNESCO, este se desarrolló con el propósito de identificar y analizar el comportamiento del factor social en estas problemáticas, ello les permitió realizar una caracterización por países haciendo clasificación entre ellos.

Por otra parte, el Informe del Desarrollo de la Educación del Siglo XXI en Colombia (MEN, 2004) presenta las leyes, técnicas e iniciativas implementadas en búsqueda del mejoramiento de la educación colombiana, en él se evidencian resultados obtenidos en el marco de esas intervenciones.

Por todo lo anterior nace la presente investigación, la cual busca caracterizar los departamentos de la región Caribe en Colombia haciendo uso de indicadores de logro educativo como lo es la asistencia escolar en los departamentos colombianos, haciendo un énfasis especial en la región Caribe.

\section{METODOLOGÍA}

Esta investigación está orientada a un diseño descriptivo de corte transversal con base poblacional, donde se caracterizarán a los 32 departamentos colombianos, haciendo especial énfasis en los pertenecientes a la región Caribe de acuerdo al índice de logro educativo (ILE) entre los cuales se encuentra la asistencia escolar por grupos de edad todo esto entre los años 2004 y 2005.

\subsection{EL MÉTODO}

El Análisis de Componentes Principales -ACP en adelante- es una técnica estadística de síntesis de la información o reducción de la dimensión, este análisis realiza dos acciones fundamentales: cuantifica las variables originales y reduce la dimensionalidad de los datos (Navarro, Casas, \& González, 2010).

\subsection{COMPONENTES DEL MÉTODO}

El ACP es una representación de tablas de datos cuantitativos la cual se compone de individuos y variables (Vertel, Botero, \& Cepeda, 2016).

\section{PROCEDIMIENTO ACP EN R}

Para del desarrollo del a investigación se utilizará el software R (2017), software estadístico libre y gratis.

En $\mathrm{R}$ para realizar ACP a una tabla de datos cuantitativa se debe contar con dos paquetes fundamentales, los cuales son FactoClass y ade4. FactoClass 
es un paquete que combina métodos factoriales con análisis de conglomerados, el cual permite aplicar técnicas de análisis multivariado. Este por medio de cada una de sus funciones presenta planos factoriales, valores propios, vectores, coordenadas y contribuciones de filas y columnas, lo cual en su conjunto ayuda a la interpretación y análisis de la información (Pardo \& Del Campo, 2007). Ade4 es una herramienta para el análisis de datos multivariados, la cual contribuye a la realización de varios métodos para el análisis de tablas de datos, como, por ejemplo, análisis de componentes principales, análisis de correspondencia, entre otros (Dray \& Dufour, 2007).

\section{ANÁLISIS Y RESULTADOS}

Para el caso del análisis de la asistencia escolar por grupos de edad de los departamentos objeto de estudio, las variables involucradas fueron evaluadas en porcentaje (\%). En la figura 1, aparecen las variables continuas activas, las cuales se utilizan en el ACP, junto con sus estadísticas básicas en la tabla 1.

Figura 1. Boxplot indicadores de logro educativo: Asistencia escolar.

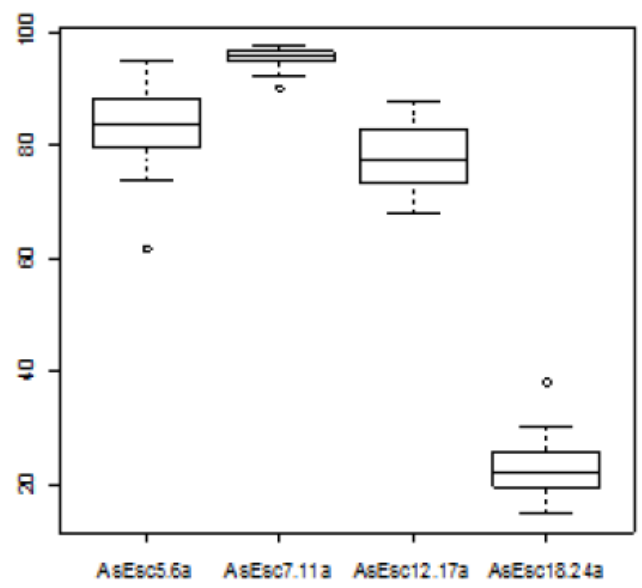

Fuente: Elaboración propia a partir de información del Departamento Nacional de Planeación.

Esta información permite evidenciar que el grupo poblacional que presenta el mayor porcentaje promedio de asistencia escolar es el grupo de 7 a 11 años con un $95.52 \%$, le sigue el grupo de 5 a 6 años con $82.67 \%$, continua el grupo de 12 a 17 años con $77.91 \%$ y por último se tiene el grupo de 18 a 24 años con el porcentaje promedio más bajo de $23.14 \%$ de asistencia escolar. 
Tabla 1.Resumen numérico indicadores de logro educativo: Asistencia escolar.

\begin{tabular}{|l|l|l|l|}
\hline \multicolumn{1}{|c|}{ AsEsc5.6a } & \multicolumn{1}{c|}{ AsEsc7.11a } & \multicolumn{1}{c|}{ AsEsc12.17a } & \multicolumn{1}{|c|}{ AsEsc18.24a } \\
\hline Min.:61.60 & \multicolumn{1}{|c|}{ Min.:90.20 } & \multicolumn{1}{c|}{ Min.:68.20 } & Min.:14.90 \\
\hline 1st Qu.:79.67 & 1st Qu.:94.88 & 1st Qu.:73.47 & 1st Qu.:19.48 \\
\hline Median:83.50 & Median:95.65 & Median:77.35 & Median:21.85 \\
\hline 3rd Qu.:87.67 & Mean:95.52 & Mean:77.91 & Mean:23.14 \\
\hline Max. :95.10 & Max. :97.60 & Mrd Qu.:82.55 & 3rd Qu.:25.75 \\
\hline
\end{tabular}

Fuente: Elaboración propia a partir de información del Departamento Nacional de Planeación.

Así mismo de los departamentos evaluados la asistencia escolar en el grupo de 5 a 6 años presenta un mínimo de 61.60\% y un máximo de 95.10, para el caso de la asistencia escolar en el grupo de 7 a 11 años esta evidencia un mínimo de $90.20 \%$ y un máximo de $97.60 \%$.

Por su parte en el grupo de 12 a 17 años el mínimo de asistencia escolar fue de $68.20 \%$ y el máximo de $87.90 \%$ y por ultimo para el grupo de 18 a 24 años el mínimo fue de 14.90 y el máximo de $37.90 \%$.

Esto evidencia que el grupo de la población de jóvenes entre los 18 y 24 años es la que presenta los menores niveles de asistencia escolar.

Por otra parte, se realizó un Análisis en componentes principales (ACP)normado-centrado, donde los dos primeros ejes factoriales explicaron más del $88.8 \%$ de la variabilidad total del estudio.

En términos de las variables se evidencia que las variables asistencia escolar para el grupo de 18 a 24 años presenta una fuerte correlación la asistencia escolar para el grupo de 12 a 17 años. Mientras que la asistencia escolar para el grupo de 5 a 6 años y el grupo de 7 a 11 años presenta una mayor correlación entre ellas. Ello muestra que para el caso de los departamentos objeto de estudio la asistencia escolar para los grupos poblacionales de menor edad se correlaciona entre ellos y los de mayor edad se correlacionan entre ellos. 


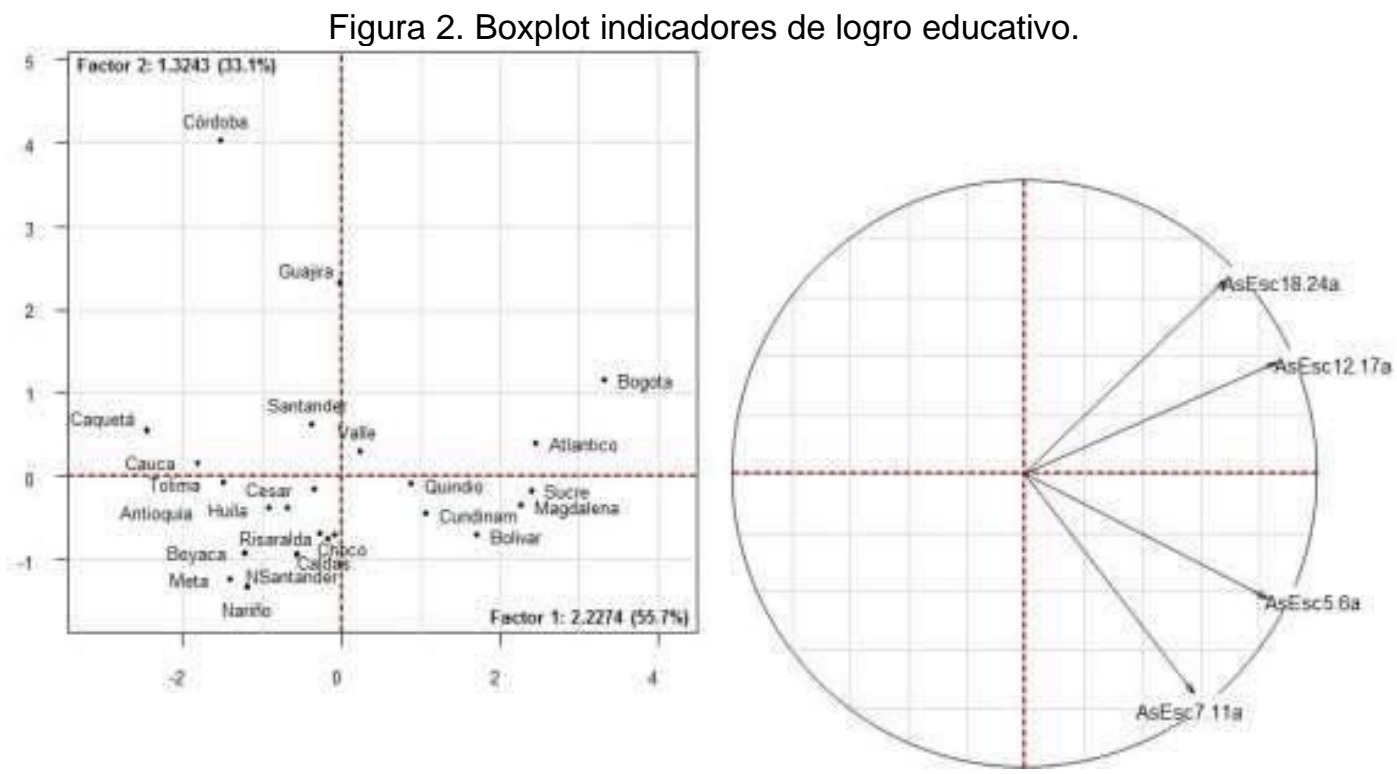

Fuente: Elaboración propia a partir de información del Departamento Nacional de Planeación.

Por otra parte, en términos de los departamentos se observa que departamentos como Quindío, Cundinamarca, Bolívar, Magdalena, Sucre, Atlántico y Bogotá D.C presentan las tasas más altas de asistencia escolar en todos los niveles; mientras que los departamentos de Caquetá, Guajira, Córdoba, Cesar, Tolima, Risaralda, Boyacá, Choco, Norte de Santander, Caldas, Choco, Huila, Meta, Risaralda y Antioquia presentan los niveles más bajos de asistencia escolar.

De la región Caribe se observa que los mejores niveles de asistencia escolar la presentan Sucre, Magdalena y Bolívar compartiendo el nivel con Bogotá y Cundinamarca.

\section{CONCLUSIONES PRINCIPALES.}

Los departamentos de Colombia presentan mejores indicadores de logros educativos en términos de la asistencia escolar son: Quindío, Cundinamarca, Bolívar, Magdalena, Sucre, Atlántico y Bogotá D.C.

En cuanto a la región Caribe se observa que los mejores niveles de asistencia escolar la presentan Sucre, Magdalena y Bolívar.

En donde el grupo de la población de jóvenes entre los 18 y 24 años es la que presenta los menores niveles de asistencia escolar. 


\section{REFERÊNCIAS}

André Lestage. Analfabetismo y alfabetización. Organización de las Naciones Unidas para la Educación, la Ciencia y la Cultura. Recuperado de http://unesdoc.unesco.org/images/0013/001339/133942so.pdf, 1981.

García, P. "Indicadores Sociales Departamentales", SISD 37. Bogotá, D. C.,Colombia. 2005.

Terrádez, M. "Análisis de componentes principales". Proyecto e-Math1 Financiado por la secretaria de Estado de Educación y Universidades (MECD). 2010

Viloria, J. "Políticas para transformar el capital humano en el Caribe colombiano". Documentos de trabajo sobre economía regional. DNP 2007. SISD 37. Bogotá, D. C., Colombia. 2006

Ministerio de Educación Nacional -MEN-. La revolución educativa 2002 2006". Informe de gestión $7 \quad$ agosto 2006. Http://www.mineducacion.gov.co/1621/articles-104306_archivo_pdf.pdf, 2006.

R Development Core Team. R: A language and environment for statistical computing, R Foundation statistical computing, Vienna, Austria. ISBN 3-90005107-0. Http://www.R-proyect.org. 2017

Dray, S., \& Dufour, A.-B.. The ade4 Package: Implementing the Duality Diagram for Ecologists. Journal of Statistical Software. 2007.

Navarro, J., Casas, G., \& González, E. . Análisis de componentes principales y análisis regresión para datos categóricos. Aplicación en la hipertensión arterial. Revista de Matemática: Teoría y Aplicaciones, 199-230. 2010.

Pardo, C., \& Del Campo, P. . Combinación de métodos factoriales y de análisis de conglomerados en R: el paquete factoclass. Revista Colombiana de Estadística, 231-245. 2007

Vertel, M., Botero, L., \& Cepeda, J. Análisis Multivariado de Datos. Sincelejo: Ediciones Universidad Simón Bolívar. 2016. 